\title{
A Comparison of Various Monoclonal Antibodies to the Previous Standard of Care Chemotherapy in the Treatment of Advanced- Stage Melanoma
}

\author{
Kevin Verde \\ Nova Southeastern University, kv259@nova.edu \\ Lauren Johnson \\ Nova Southeastern University, lj704@nova.edu \\ Alex Clancy \\ Nova Southeastern University, ac2295@nova.edu \\ Ashley Goldberg \\ Nova Southeastern University, ag1632@nova.edu \\ Aleia Monden \\ Nova Southeastern University, am2903@nova.edu
}

See next page for additional authors

Follow this and additional works at: https://nsuworks.nova.edu/ijahsp

Part of the Dermatology Commons, Family Medicine Commons, Internal Medicine Commons, and the Other Medical Specialties Commons

\section{Recommended Citation}

Verde K, Johnson L, Clancy A, Goldberg A, Monden A, Philip P. A Comparison of Various Monoclonal Antibodies to the Previous Standard of Care Chemotherapy in the Treatment of Advanced-Stage Melanoma. The Internet Journal of Allied Health Sciences and Practice. 2016 Jan 01;14(3), Article 14.

This Manuscript is brought to you for free and open access by the College of Health Care Sciences at NSUWorks. It has been accepted for inclusion in Internet Journal of Allied Health Sciences and Practice by an authorized editor of NSUWorks. For more information, please contact nsuworks@nova.edu. 


\title{
A Comparison of Various Monoclonal Antibodies to the Previous Standard of Care Chemotherapy in the Treatment of Advanced-Stage Melanoma
}

\begin{abstract}
In the year 2015, it is estimated that the number of new cases of invasive melanoma will be 42,670 in males

and 31,200 in females. ${ }^{1}$ Melanoma is treatable with early diagnosis; however, more advanced disease has devastating outcomes. For the past decade, two chemotherapy agents, dacarbazine and temozolomide, have been the treatment of choice for advanced stage III or IV melanoma requiring systemic treatment. Interleukin-2 (IL-2) therapy has been used but with serious side effects. More recently, the focus has shifted to monoclonal antibodies and enzyme inhibitors as the main systemic treatment for advanced cutaneous melanoma. This literature review gathered several studies which looked at the use of monoclonal antibodies, and compared monoclonal antibodies to conventional chemotherapy to assess whether there is a significant difference in tumor response, sustained remissions and side effect profile. An extensive medical literature review was conducted with PubMed and Cochrane databases using the keywords: "monoclonal antibody," "melanoma," and "treatment." This list of articles was further narrowed by specific inclusion and exclusion criteria as well as reviewed for validity and quality using the GRADE system. Seven clinical trials were included in this literature review. One observational study evaluated the overall safety and efficacy of monoclonal antibodies, while another compared monoclonal antibodies versus placebo under the same variables. Three of the research studies were randomized clinical trials evaluating the safety and efficacy of monoclonal antibodies in comparison to chemotherapy. Two retrospective studies assessed patients from expanded access programs who did not meet criteria to participate in a clinical trial. All seven studies had similar inclusion and exclusion criteria and the patients were prognostically similar before starting treatment. Six out of the seven studies demonstrated superiority of monoclonal antibodies advanced-stage melanoma treatment. One study failed to demonstrate a statistically significant survival advantage over traditional chemotherapy. The use of monoclonal antibodies has been demonstrated to be a more specific and effective treatment approach than other therapies tried in the past. While monoclonal antibodies have demonstrated efficacy in first line treatment for advanced stage melanoma, further research is necessary to determine which combination of medications is most beneficial for these patients.
\end{abstract}

\section{Author Bio(s)}

Lauren Johnson, currently a second year Physician Assistant student at Nova Southeastern University, received her Bachelor's in Psychology in 2012 from Geneseo State University where she co-authored a study published in the Journal of Neurotoxicology and Teratology. Lauren grew up in New York and resides in Florida with her family.

Kevin Verde is a second year Physician Assistant student at Nova Southeastern University. He obtained his Bachelor's in Economics from Florida International University. Kevin worked as a Clinical Research Coordinator at the Holy Cross Orthopedic Institute in Fort Lauderdale, Fl. He is a trumpet player and admirer of the arts.

Alex Clancy is currently in her second year at Nova Southeastern University's Physician Assistant Program. She was born and raised in Southern California and obtained her Bachelors Degree at the University of San Diego in 2012. She plans to pursue a residency in emergency medicine or work in urgent care.

Aleia Monden is a Physician Assistant Student at Nova Southeastern University. She is interested in Dermatology and Pediatric Orthopedic Surgery. Aleia attended Florida State University when she became an Olympic Trial qualifier, and five-time USA Diving National Finalist for Platform Diving and obtained her Bachelor's Degree in Exercise Science. 
Ashley Goldberg, currently a second year Physician Assistant student at Nova Southeastern University, is interested in pursuing a career in dermatology. She graduated from the University of Maryland with a Bachelor's in Community and Behavioral Health. She hopes to one day pursue her culinary passion and open a restaurant.

Priya Philip, currently a second year Physician Assistant student at Nova Southeastern University, received her Bachelor's in Biology from the same institution. She holds an Associate Degree in Nuclear Medicine Technology and worked as a Nuclear Medicine Technologist for seven years. She resides in Florida with her husband and son.

\section{Acknowledgements}

Many thanks to our faculty adviser J. Keith Williams MPAS, PA-C for his guidance throughout the process of writing this paper.

\section{Authors}

Kevin Verde, Lauren Johnson, Alex Clancy, Ashley Goldberg, Aleia Monden, and Priya Philip 


\section{A Literature Review of the Comparison of Various Monoclonal Antibodies to the Previous Standard of Care Chemotherapy in the Treatment of Advanced-Stage Melanoma}

\section{BACKGROUND}

The American Cancer Society estimates that in 2012 there were 14.1 million new diagnoses of cancer made worldwide. Out of the 14.1 million new cases, 8.2 million succumbed to their disease. ${ }^{1}$ Cancers of the skin are the most common. ${ }^{2}$ Melanoma, a cancer of the skin cells called melanocytes, accounts for only $2 \%$ of these skin cancers, but causes the majority of skin cancer deaths. In 2015 , it is estimated that 73,870 new melanoma diagnoses will be made, and 9,940 of those patients will pass away. ${ }^{2}$

Melanoma is staged in a four-tier system. Each stage has one to three sub-stages. Generally, the 5 and 10 year survival rate decreases as the stages increase, the one exception being stage IIC versus stage IIIA and IIIB. ${ }^{3}$ The 5 year survival rate for a patient with stage I disease is $97 \%$ compared to the 5 year survival rate for a patient with stage IV disease, which is $15 \%$ to $20 \%{ }^{3}$ With this drastic difference in the death rate between stage I and IV, it is imperative that clinicians take a look at treatment options, especially those available to patients with advanced-stage disease.

The treatment for malignant melanoma is dependent on the stage of disease. Generally, in early disease states and patients with limited metastases, surgical resection is the treatment of choice. If diagnosed early, surgery is often curative. Once the cancer reaches stage IV, surgery is no longer an immediate and definitive option, and as a result the majority of patients require systemic therapy. The history of systemic therapy for advanced cutaneous melanoma is disappointing. The treatments available have not been demonstrated to provide patients with a lasting remission, and many of the side effects were toxic and often times unbearable.

Two chemotherapeutic drugs, dacarbazine and temozolomide, were the predominant go-to systemic treatment for patients with metastatic melanoma in the past. Dacarbazine is considered to be the most active single-agent chemotherapy for patients with advanced melanoma. The response rate is anywhere between $8 \%$ to $20 \%$, but none of the responses are complete and lasting. ${ }^{4}$ Temozolomide is an analog of dacarbazine. Unlike dacarbazine, temozolomide is absorbed orally and crosses the blood brain barrier, so it was thought to have better response rates than dacarbazine. ${ }^{5}$ When compared directly to dacarbazine, temozolomide shows no significant improvements in overall survival. ${ }^{6}$

High-dose interleukin-2 (IL-2) was the first immunotherapy used for advanced stage melanoma which provided patients with a durable remission. ${ }^{7}$ Interleukin-2 is a T-cell growth factor that was discovered in the late 1970s. After its discovery, it was widely used as a treatment for advanced-stage cancers of multiple origins, and especially those who failed first line treatment options. It soon became the drug of choice in advanced-stage melanoma treatment. Although this was the first treatment discovered that demonstrated a significant response for patients with stage IV melanoma, it came with significant drawbacks. IL-2's severe multi-organ toxicity limited the number and type of patients who were approved to use the treatment. IL-2 also requires that treatment, if given, must be administered by specific physicians at hospitals equipped to handle the potential side effects. In addition, the same efficacy was not seen in patients who used IL-2 after previous systemic therapy. As a result, many patients were forced to use cytotoxic chemotherapy, which demonstrates no clinical benefit and only provides palliative care.

As a result of the limited use of IL-2 and the poor response to cytotoxic chemotherapy, more treatment options have been sought for advanced-stage melanoma. Targeted therapies using monoclonal antibodies and enzyme inhibitors have now made their way to the forefront of systemic treatment for advanced cutaneous melanoma. Monoclonal antibodies are antibodies that are directed towards specific receptors and ligands on the surface of cells, which when binding, prevent cellular activation. Enzyme inhibitors are molecules that bind directly to specific enzymes which then act as competitive inhibitors of ATP binding. ${ }^{9}$ These treatments, when used alone or in combination with one another or chemotherapy, provide patients with a treatment approach that is much more precise than previous treatments were able to accomplish in the past, and are said to be more tolerable than previous therapy with cytotoxic chemotherapy and/or IL-2.

In the case of melanoma treatment, there are 4 pathways that are targeted with immunotherapy: checkpoint inhibition with the anti-PD1 antibody (pembrolizumab, nivolumab), checkpoint inhibition with the anti-CTLA4 antibody (ipilimumab), BRAF gene inhibition (vemurafenib, dabrafenib) and MEK gene inhibition (trametinib, cobimetinib). Checkpoint inhibition with the anti-PD1 antibody used in combination with the CTLA-4 antibody is now the preferred 
treatment for advanced stage melanoma. ${ }^{8}$ The purpose of this literature review is to compare various monoclonal antibodies when used alone, to the previous standard of care chemotherapy to see if there is a difference in tumor response, durable remissions and patient tolerability.

\section{METHODS}

The PubMed and Cochrane databases were searched using the keywords "monoclonal antibody," "melanoma," and "treatment." PubMed yielded 2,835 articles and Cochrane yielded 33 results. Articles written within the last 5 years were extracted for further review. Titles and abstracts were read for relevance and articles discussing the efficacy and safety of monoclonal antibody use in the treatment of melanoma and those comparing the use of monoclonal antibody therapy with standard chemotherapy were further reviewed. Twenty articles from PubMed's database and 5 articles from Cochrane's database were eligible for more extensive review. These 25 articles were read in more detail to ensure that they met the following inclusion/exclusion criteria.

Inclusion Criteria

1. Articles written in English.

2. Articles published between 2010 and 2015.

3. Clinical trials evaluating the safety and efficacy of monoclonal antibodies as treatment for Stage III melanoma, Stage IV melanoma, and recurrent melanoma.

4. Clinical trials evaluating the safety and efficacy of monoclonal antibodies directly compared with standard chemotherapy.

5. Retrospective studies specifically published after evaluation of use of monoclonal antibodies in treatment of melanoma as part of an expanded access program (EAP) to evaluate the use of this treatment option in a typical clinical setting.

\section{Exclusion Criteria}

1. Articles written before 2010.

2. Articles written in a language other than English.

3. Clinical trials completed in non-human subjects.

4. Clinical trials where monoclonal antibodies were used in combination with other drugs.

5. Case studies.

6. Trials evaluating ranges of dosing for treatment.

7. Retrospective studies that were not conducted as part of an expanded access program (EAP).

8. Literature Reviews.

9. Clinical trials that evaluated the use of monoclonal antibodies for treatment of cancers other than melanoma.

\section{RESULTS}

Twenty-five articles were reviewed and evaluated based on our inclusion/exclusion criteria and standard critical appraisal guideline (CASP). Only articles discussing clinical trials and retrospective studies conducted with expanded access programs were included. Seven articles met our criteria for inclusion into this review. Four of the articles discuss the safety and efficacy of specific drugs within the class of monoclonal antibodies. The other 3 articles directly compared the efficacy of a drug within the class of monoclonal antibodies to the standard chemotherapy of choice. Articles were evaluated using the GRADE criteria for validity and quality. 


\begin{tabular}{|c|c|c|c|c|c|c|c|}
\hline \multicolumn{8}{|c|}{ Table 1. GRADE Profile } \\
\hline Author & $\begin{array}{l}\text { Participants } \\
\text { (Studies) }\end{array}$ & $\begin{array}{l}\text { Risk of } \\
\text { Bias }\end{array}$ & Inconsistency & Indirectness & Imprecision & $\begin{array}{c}\text { Publication } \\
\text { Bias }\end{array}$ & $\begin{array}{c}\text { Overall } \\
\text { Quality of } \\
\text { Evidence }\end{array}$ \\
\hline Kirkwood et al & $251($ OS)a & Serious ${ }^{b}$ & Not Serious & Not Serious & Not Serious & None & $\begin{array}{c}\text { Very Low } \\
+\end{array}$ \\
\hline $\begin{array}{c}\text { Eggermont et } \\
\text { al }\end{array}$ & 951 (RCT) & Serious $^{\mathrm{C}}$ & Not Serious & Not Serious & Not Serious & None & $\begin{array}{c}\text { Moderate } \\
+++\end{array}$ \\
\hline $\begin{array}{c}\text { Altomonte et } \\
\text { al }\end{array}$ & $74(\mathrm{OS})^{\mathrm{a}}$ & Serious $^{d}$ & Not Serious & Not Serious & Not Serious & None & $\begin{array}{c}\text { Very Low } \\
+\end{array}$ \\
\hline $\begin{array}{c}\text { Di Giacomo et } \\
\text { al }\end{array}$ & $27(\mathrm{OS})^{\mathrm{a}}$ & $\begin{array}{c}\text { Not } \\
\text { Serious }\end{array}$ & Not Serious & Not Serious & Seriouse & None & $\begin{array}{c}\text { Very Low } \\
+\end{array}$ \\
\hline Robert et al & 418 (RCT) & $\begin{array}{c}\text { Not } \\
\text { Serious }\end{array}$ & Not Serious ${ }^{f}$ & Not Serious & Not Serious & None & $\begin{array}{c}\text { High } \\
++++\end{array}$ \\
\hline Weber et al & 405 (RCT) & Serious ${ }^{9}$ & Not Serious & Not Serious & Not Serious & None & $\begin{array}{c}\text { Moderate } \\
+++\end{array}$ \\
\hline Ribas et al & 655 (RCT) & Serioush & Seriousi & Not Serious & Not Serious & None & $\begin{array}{c}\text { Low } \\
++\end{array}$ \\
\hline
\end{tabular}

OS = Observational Study RCT = Randomized Controlled Trial

\section{Overall Quality Among All Studies: Low}

aAll Observational Studies automatically start at a "low" overall quality of evidence.

bSome of the authors reported being either being employed, having ownership interests in, or receiving funding from Pfizer, Intrexon, Schering, Vical, Bristol-Myers Squibb, Glaxo, Wyeth, Novartis, Genentech and Johnson \& Johnson.

'Some of the authors reported receiving personal fees, grants, holding leadership roles, being employed, and/or providing other services to Bristol-Myers Squibb, Amgen, Merck, Medlmmune, GlaxoSmithKline, Roche, Novartis and Merck Sharp and Dohme. dSome of the authors reported holding leadership roles and/or receiving funding from Bristol-Myers Squibb, Merck Sharp and Dohme, Roche-Genentech, GlaxoSmithKline, Amgen, and Celgene.

eStudy had a small population size of 27 patients.

fFour patients were unintentionally enrolled despite having an ECOG status of 2. One additional patient was enrolled without reporting an ECOG status.

iSome of the authors have reported receiving grants, funding, honoraria, personal fees, holding leadership roles, owning stock and/or being employees of Bristol-Myers Squibb, Merck Sharp and Dohme, Genentech, Astrazeneca, AbbVie, GlaxoSmithKline, Macrogenics, Ichor Therapeutics, Lion Biotechnologies, Pierisl, Celldex Therapeutics, Altor BioScience, cCAM Biotherapeutics, Novartis, Merck Serono, Almirall-Hermal, Leo Pharma, Amgen, Galderma, Janssen, Boehringer Ingelheim, Pfizer, Provectus, Prometheus, National Comprehensive Cancer Network, Celgen, Genentech, Threshold, Eisai, Roche, Allos, AstraZeneca Medlmmune, Anaeropharma, Merus, Symphogen, Nektar, Kyowa-Kirin, Astellas-Agensys, Amphivena, Neostem, Seattle Genetics, Immunova, TRM Oncology, Physicians Education Resource, Imedex, Research to Practice, Ventana, Lilly, One author has a pending patent from Immune Target.

hSome of the authors indicated a financial or other interest relevant to the subject matter. They either held leadership roles, owned stock, were employed by and received honoraria or research funding from Pfizer, Amgen, Bristol-Myers Squibb, Genentech, GlaxoSmithKline, Millenium Pharmaceuticals, Novartis, Genta, Merck Sharp and Dohme, Philogen, Roche, SOBI, Plexikkon, Celgene, Eisai.

An unknown number of patients received ipilimumab at other sites when they were only being treated with Tremelimumab as part of the study.

Kirkwood et al saw the need for new treatment options in patients with metastatic melanoma, as current therapies including interleukin-2, chemotherapy with dacarbazine or temozolomide and IFN-a2b yield low responses with high toxicity..$^{10}$ As such, Kirkwood et al. ${ }^{10}$ conducted a multicenter, phase II, single-arm study in order to assess the objective rate response rate of tremelimumab, a fully human lgG2 monoclonal antibody specific for CTL-associated antigen 4 (CTLA-4), as well as calculate the safety profile and pharmacokinetics. Kirkwood et al. ${ }^{10}$ specifically looked at the best overall response to the drug, while secondary endpoints included safety and tolerability to the drug, durable response rate, overall survival, duration of tumor response, and progression-free survival. Tremelimumab was administered using $15 \mathrm{mg} / \mathrm{kg}$ every 90 days or $10 \mathrm{mg} / \mathrm{kg}$ every month. Tumor assessments were completed at the end of each cycle and on day 60 of the second cycle. If a tumor response was present after 6 months, this was defined as 
a durable response. Patients 18 years and older with surgically incurable Stage III or IV melanoma that was refractory, or had relapsed after one or more cycle of prior treatment with IL-2, dacarbazine, temozolomide, or IFN-a for metastatic melanoma were considered eligible. The study consisted of 251 patients between December 2005 and November 2006. Participants had to meet the above inclusion and exclusion criteria with the addition of having one or more measurable lesions according to Response Evaluation Criteria in Solid Tumors (RECIST) guidelines, "serum lactate dehydrogenase (LDH) level $\leq 2 \times$ upper limit of normal (ULN) and adequate bone marrow, hepatic, and renal function determined within 14 days of enrollment (absolute neutrophil count $\geq 1.5 \times 109$ cells $/ \mathrm{L}$, platelets $\geq 100 \times 109$ cells/L, hemoglobin $\geq 10 \mathrm{~g} / \mathrm{dL}$, aspartate aminotransferase and alanine aminotransferase $\leq 2.5$ or $\leq 5 \times$ ULN with liver metastases, total bilirubin $\leq 2 \times U L N$, and serum creatinine $\leq 2.0 \mathrm{mg} / \mathrm{dL}$ ). Additionally, patients were required to have recovered from all previous treatment-related toxicities to baseline status or to grade 0 or 1 according to National Cancer Institute Common Terminology Criteria for Adverse Events version 3.0."10

Additionally, patients were excluded if they had previously been treated with any CTLA4-inhibiting agent, had any treatment for cancer within one month of enrolling into the trial, a history of autoimmune disease, inflammatory bowel disease, any vaccine therapy for melanoma within six months of enrollment, a potential need for systemic corticosteroids or concurrent immunosuppressive drugs, brain metastases (unless no longer detectable after previous treatment), and a history of other malignancies not including basal cell carcinoma, squamous cell carcinoma, or cervical carcinoma. ${ }^{10}$

Therapy was discontinued if there was disease progression, or if toxicity occurred and the side-effects were considered intolerable. After each cycle, a subsequent dose was only administered if the patient had an adequate performance status, hepatic function, renal function, and acceptable amylase and lipase levels. Additionally, subsequent dosing was only administered if treatment-related adverse effects were resolved and considered tolerable by the day the dose was to be administered. Most (77\%) of patients developed a treatment-related adverse effect of any grade, however most were mild-moderate, (grade 1 or 2). Fifty-one (21\%) patients developed adverse effects greater than grade 2. Fortyseven (19\%) patients had more serious adverse effects. Some of the common adverse effects included nausea, diarrhea, fatigue, vomiting, and pruritus. Thirteen patients discontinued the study due to treatment-related adverse effects and two treatment-related deaths occurred. In nine patients, treatment-related endocrine adverse effects occurred, with eight of these patients obtaining thyroid disorders, and one patient with hypophysitis and pituitary insufficiency. ${ }^{10}$

The results of the study by Kirkwood et al revealed that the average overall survival for all participants was 10 months, while the clinical overall rate, that is, the overall response with stable disease, was $21 \% .10$ All lesions were measured according to RECIST guidelines. Two-hundred and forty-six patients received at least one dose of tremelimumab. On average, participants received one dose, while 109 patients (44\%) received two or more doses. Sixteen (6.6\%) patients had partial responses, 35 reached a stable disease state (defined as no disease progression for at least 70 days following initial dosing), three achieved responses within the first cycle, and no responders achieved complete response. All those that responded to treatment sustained the response for at least 170 days after enrollment and as such, met the criteria for a "durable response." Eleven patients had ongoing responses at the time of the last tumor assessment, one patient with a family history of sudden death died of a cardiac event 321 days after enrollment, while 15 patients that responded to treatment are still currently alive with overall safety ranging from 20.1 to 34.1 months. Eight (3.3\%) patients achieved responses in target lesions, despite progressive disease within the first cycle, and all eight survived for $>20$ months. Progression-free survival was $15 \%$ at six months, $40.3 \%$ at 12 months, and $22 \%$ at 24 months. Baseline Eastern Cooperative Oncology Group (ECOG) status was the strongest indicator for survival, while age and sex did not seem to strongly affect survival. The study showed no difference in overall survival between patients that previously received interferon or previous IL-2, versus those with no prior interferon, contrary to prior studies, which indicated a significant shorter time to progression in those previously receiving IL-2.10 


\begin{tabular}{|c|c|c|c|c|c|}
\hline & $\begin{array}{c}\text { Primary } \\
\text { Endpoint }\end{array}$ & & Secondary & points & \\
\hline & $\begin{array}{l}\text { Overall } \\
\text { response }\end{array}$ & $\begin{array}{l}\text { Duration of tumor } \\
\text { response }\end{array}$ & Overall survival & $\begin{array}{c}\text { Progression free } \\
\text { survival }\end{array}$ & Safety \\
\hline Tremelimumab & $6.6 \%$ & $\begin{array}{c}8.9 \text { to } 28.9 \\
\text { months }\end{array}$ & 10 months & $\begin{array}{l}15.0 \% \\
40.3 \% \\
22.0 \%\end{array}$ & $77 \%$ \\
\hline
\end{tabular}

Progression free survival: patient's disease reaches a plateau and no longer progresses. Measured $15 \%$ at 6 months, $40.3 \%$ at 12 months, $22 \%$ at 24 months

Safety: percent of patients who experienced an adverse event. Most adverse events were mild to moderate (grade 1/2). Grade $3 / 4$ adverse events included diarrhea (11\%), fatigue (2\%), and colitis (4\%).

Eggermont et al conducted a multinational, randomized, double-blind phase III trial evaluating patients with histologically confirmed melanoma metastatic only to local lymph nodes between July 10, 2008 and Aug 1, 2011.11 The study primarily looked at recurrence-free survival in patients with primary cutaneous stage III melanoma that had been completely excised with adequate surgical margins and complete regional lymphadenectomy at least 12 weeks prior to randomization. Trials were performed in 91 hospitals across 19 countries and consisted of a total of 951 patients who were randomly assigned to treatment with ipilimumab, a fully human monoclonal antibody that augments anti-tumor immune responses by blocking CTLA-4, or a placebo. The patient population included those age 18 or older with stage IIIA, stage IIIB, or stage IIC melanoma with no metastasis present at time of selection into the trial. Patients had to meet the above inclusion criteria in addition to an Eastern Cooperative Oncology Group: performance status less than 1 , with no cardiovascular disease, autoimmune disease, uncontrolled infections, white blood cell count lower than $2.5 \times$ 109 cells per L, absolute neutrophil count lower than $1.0 \times 109$ cells per L, platelets lower than $75 \times 109$ cells per $\mathrm{L}$, hemoglobin concentration less than $9 \mathrm{~g} / \mathrm{dL}$, creatinine higher than 2.5 times the upper normal limit, hepatic enzymes or lactate dehydrogenase higher than two times the upper normal limit, use of systemic corticosteroids, and previous systemic therapy for melanoma. ${ }^{11}$

Random assignments were stratified by disease stage and regions of the world including North America, Europe, and Australia. The patients received either a placebo or IV infusions of ipilimumab $10 \mathrm{mg} / \mathrm{kg}$ every three weeks for four doses, then every three months for up to a maximum of three years, or until unacceptable toxicity, major protocol violation, disease recurrence, or the patient refused treatment. The initial four doses were considered induction doses, additional doses thereafter were considered maintenance doses and were added based on principles of re-stimulation of the immune system. ${ }^{11}$

The result of the Eggermont et al study showed that the median recurrence-free survival was appreciably longer in the ipilimumab group at 26.1 months versus the placebo group at 17.1 months. ${ }^{11}$ Three-year survival was also expectedly longer for the ipilimumab group at $46.5 \%$ versus the $34.8 \%$ in the placebo group. In those with stage IIIC disease, ipilimumab's effect was the most evident. The average number of doses received per patient was four in the ipilimumab group and eight in the placebo group. The primary goal of the study was recurrence-free survival which was defined as the time between the date of randomization and the date of initial recurrence or death. "Of 471 patients who started ipilimumab, $245(52 \%)$ discontinued treatment because of an adverse event, of which $230(49 \%)$ were drug related; in $182(39 \%)$ patients the adverse event leading to discontinuation happened within 12 weeks of the start of treatment."11 The most common adverse effects were gastrointestinal, hepatic, and endocrine, in which most patients had only one serious episodic event. In the ipilimumab group, a total of five patients died due to drug-related adverse effects including one due to myocarditis, one due to multi-organ failure with Guillain-Barre syndrome, and three patients died due to colitis. There were no deaths related directly to treatment in the placebo group. As a whole, the authors found that the drug, ipilimumab, showed a significantly increased recurrence-free survival in patients with highrisk stage III melanoma that had been completely resected. ${ }^{11}$ 
Table 3. Adjuvant ipilimumab versus placebo after complete resection of high-risk stage III melanoma11

\begin{tabular}{|c|c|c|c|c|c|}
\hline & \multirow{2}{*}{$\begin{array}{c}\begin{array}{c}\text { Primary } \\
\text { Endpoint }\end{array} \\
\begin{array}{c}\text { Recurrence free } \\
\text { survival }\end{array}\end{array}$} & \multicolumn{4}{|c|}{ Secondary Endpoints } \\
\hline & & $\begin{array}{l}\text { Distant metastasis } \\
\text { free survival }\end{array}$ & Overall survival & $\begin{array}{l}\text { Adverse event } \\
\text { profile }\end{array}$ & $\begin{array}{l}\text { Health related } \\
\text { quality of life }\end{array}$ \\
\hline Ipilimumab & 26.1 & $p$ & $p$ & $99 \%$ & $p$ \\
\hline Placebo & 17.1 & $p$ & $p$ & $91 \%$ & $p$ \\
\hline
\end{tabular}

Recurrence free survival: patients still alive after start of treatment and without disease

Distant metastasis free survival: patients still alive after start of treatment without distant metastasis

Adverse event: any mild to severe side effect that occurred after the start of the study

*p=pending

Altomonte et al conducted a randomized controlled phase III trial involving ipilimumab. Researchers used data from an expanded access program in Italy. ${ }^{12}$ The program evaluated clinical activity and safety of the monoclonal antibody in patients with advanced melanoma disease in a daily practice setting. Clinical activity and safety profile of ipilimumab was evaluated in patients older than 16 years of age with advanced melanoma in a setting similar to daily practice. Patients were eligible for participation in the expanded access program if they had histologically confirmed unresectable stage III/ IV skin, ocular, or mucosal melanoma. Patients had to previously fail systemic therapy, been intolerant to more than 1 systemic therapy, or have no other therapeutic options available. Eastern Cooperative Oncology Group (ECOG) performance status of 0, 1, or 2 was required, and at least 28 days since previous treatment with chemotherapy, biochemotherapy, surgery, radiation, or immunotherapy was recommended. Patients with asymptomatic brain metastases were included. Exclusion criteria included any other systemic therapy for the treatment of melanoma, concomitant autoimmune diseases or other malignancies, and known HIV, hepatitis B or C infection. ${ }^{12}$

Treatment in this study comprised ipilimumab $10 \mathrm{mg} / \mathrm{kg}$ every three weeks for a total of four doses. Maintenance therapy was provided every 12 weeks, if patients would continue to benefit from the treatment. Tumor responses were assessed every 12 weeks using modified World Health Organization (WHO) criteria. Seventy-four pretreated patients with advanced melanoma were treated with ipilimumab $10 \mathrm{mg} / \mathrm{kg}$. Of these patients, $43(58.1 \%)$ received all 4 induction doses, $14(18.9 \%)$ received three doses, five (6.8\%) received two doses and $12(16.2 \%)$ received only one dose. Reasons of incomplete treatment regimens include: disease progression $(n=13)$, death due to disease progression $(n=10)$, loss to follow up ( $n=5)$, drug toxicity $(n=2)$ and other unknown causes $(n=1)$. Twenty-six patients receive maintenance therapy with ipilimumab. Patients who did not receive maintenance therapy did so for the following reasons: disease progression $(n=30)$, death due to progression $(n=9)$, loss to follow-up $(n=5)$, toxicity $(n=2)$, grade II nausea and diarrhea $(n=1)$, and grade I diarrhea $(n=1)$. Of the 74 patients who started the trial, 69 were evaluated for tumor response. At week 12 of treatment, six patients had an objective response (8.7\%). At greater than 12 weeks, nine out of the 74 pretreated patients (13.0\%) had an objective tumor response, with three patients having a complete response and six having a partial response. Twenty-two patients, (31.9\%) gained disease control as defined by stable disease lasting for at least 24 weeks following the first dose of ipilimumab, complete response to treatment or partial response to treatment. Median overall survival of the total patients totaled to about 7.0 months ( $95 \%$ confidence interval, 5.3-8.7) and $16.6 \%$ of patients remained living after three years. 45 patients $(60.8 \%)$ claimed that they experienced adverse effects related to the treatment overall. Side effects included low-grade pruritus, pain, fever and diarrhea (grade I and II). Higher-grade related side effects were reported in only eight patients (10.8\%). One patient reported fever, epigastric pain, elevated AST and pancytopenia which was resolved with discontinuation of ipilimumab and use of immunosuppressive therapy, immunoglobulins and supportive therapy. ${ }^{12}$ 
Table 4. Clinical experience with ipilimumab $10 \mathrm{mg} / \mathrm{kg}$ in patients with melanoma treated at Italian centres as part of a European expanded access programme ${ }^{12}$

\begin{tabular}{lccccc}
\hline \multicolumn{7}{c}{ Measurements $^{*}$} \\
\hline $\begin{array}{c}\text { Objective } \\
\text { response }\end{array}$ & Disease Control & Overall survival & $\begin{array}{c}\text { Adverse event } \\
\text { profile }\end{array}$ & $\begin{array}{c}\text { Progression free } \\
\text { survival }\end{array}$ \\
\hline Ipilimumab & 13.0 & $31.9 \%$ & 7 months & $60.8 \%$ & 3 months
\end{tabular}

${ }^{*}$ Note: primary and secondary endpoints were not defined in this study

Objective response: any positive response to treatment after 12 weeks, of the $13 \%$ of patients: $4.3 \%$ had a complete response and $8.7 \%$ had a partial response.

Disease Control: stable disease lasting for at least 24 weeks following the first dose of ipilimumab, with complete response to treatment or partial response to treatment.

Adverse event: any mild to severe side effect that occurred after the start of the study. Most commonly grade $1 / 2$ including low-grade pruritus, pain, fever, and diarrhea. Only $10.8 \%$ were Grade $3 / 4$ adverse events.

Progression-free survival: patient's disease reaches a plateau and no longer progresses.

Di Giacomo et al completed a similar study involving ipilimumab that aimed to evaluate the feasibility of the monoclonal antibody in the treatment of metastatic melanoma in a setting similar to daily practice. ${ }^{13}$ The monoclonal antibody was utilized in an expanded access program only for patients with life-threatening, unresectable stage III/IV skin, ocular, or mucosal melanoma who had failed prior treatments with chemotherapy or for patients who did not have any other treatment options available. Patients with brain metastases were also allowed to be included in the study. Additionally, an Eastern Cooperative Oncology Group performance status of 0,1 , or 2 was required. This study also recommended at least 28 days of absence from chemotherapy, biochemotherapy, surgery, radiation, and immunotherapy. Exclusion criteria involved any other systemic therapy for melanoma, concomitant autoimmune diseases or other malignancies, and any known HIV or Hepatitis B or C infection. ${ }^{13}$

Treatment in this study included four doses of $10 \mathrm{mg} / \mathrm{kg}$ ipilimumab administered intravenously every eight weeks. Maintenance doses were provided every 12 weeks if needed. Tumors were assessed at baseline, week 12, and every 12 weeks according to WHO tumor response criteria. Disease control was defined as stable disease lasting for at least 24 weeks following the first dose of ipilimumab, complete response to treatment or partial response to treatment. Disease control rates were analyzed at 24 and 60 weeks. The rates were $29.6 \%$ at week 24 and $15 \%$ at week 60 . Median follow up was performed at 8.5 months and the overall survival rate was found to be nine months. Survival rates were established after one year $(34.8 \%)$ and two years $(23.5 \%)$. During ipilimumab treatment, lymphocyte counts greater than $1500 \mathrm{cells} / \mathrm{\mu L}$ correlated with increased overall survival (41 weeks overall survival) versus patients with lymphocyte counts less than 1500 cells/ $\mu \mathrm{L}$ (21 weeks overall survival). ${ }^{13}$

Patients receiving treatment experienced immune-related side effects that were easily managed and sometimes reversed under special circumstances. Immune-related adverse events consisted mainly of rash, pruritus, fever and diarrhea and were mainly grade 1 or 2, except for two patients which experienced grade 3 diarrhea that was reversed with antidiarrheals and corticosteroids. Two patients did not complete treatment. One was due to progression of disease and the other case was due to diarrhea upon restarting ipilimumab. One patient had a grade 3 adverse event consisting of elevated ALT/AST that resolved with steroids and allowed the patient to restart maintenance. One grade 4 adverse event related to the drug was central pancytopenia during the maintenance phase which led to discontinuation of ipilimumab; this condition was irreversible despite management resulting in death. This patient also had very rapid disease progression with metastases to the brain, lungs and soft tissue, which possibly contributed to their exiting the study. ${ }^{13}$ 
Table 5. Ipilimumab experience in heavily pretreated patients with melanoma in an expanded access program at the University Hospital of Siena (Italy). ${ }^{13}$

\begin{tabular}{|c|c|c|c|c|}
\hline & \multicolumn{4}{|c|}{ Measurements } \\
\hline & $\begin{array}{l}\text { Duration of tumor } \\
\text { response }\end{array}$ & Disease Control & Overall survival & Adverse event profile \\
\hline Ipilimumab & $7.3-32 \mathrm{mos}$ & $29.6 \%$ & 9.6 months & $65 \%$ \\
\hline \multicolumn{5}{|c|}{ Note: primary and secondary endpoints were not defined in this study } \\
\hline \multicolumn{5}{|c|}{$\begin{array}{l}\text { Disease Control: stable disease lasting for at least } 24 \text { weeks following the first dose of ipilimumab, with complete response to } \\
\text { treatment or partial response to treatment. }\end{array}$} \\
\hline \multicolumn{5}{|c|}{$\begin{array}{l}\text { Adverse event: any mild to severe side effect that occurred after the start of the study. Most common were grade } 1 / 2 \text { immune } \\
\text { related events including pruritus, fever, diarrhea. Two patients experienced grade } 3 \text { diarrhea, One patient with two week } \\
\text { elevation of AST/ALT liver enzymes. One patient experienced grade } 4 \text { pancytopenia. }\end{array}$} \\
\hline
\end{tabular}

Robert et al conducted a phase III controlled study in which they assessed the use of nivolumab, compared to chemotherapy, in treating patients with ipilimumab-refractory metastatic melanoma. ${ }^{14}$ The inclusion criteria were patients of 18 years or more with confirmed, metastatic, stage III/IV melanoma without a BRAF mutation that has not been treated in the past, and available tissue from the unresectable site. The exclusion criteria included metastases of the brain, uveal melanoma, previous melanoma treatment, and history of autoimmune disease. After randomization, there were 210 patients in the nivolumab group and 208 participants in the dacarbazine group. During database lock, $46.1 \%$ of patients receiving nivolumab and $6.3 \%$ of patients receiving dacarbazine were involved in the study. Patients in the nivolumab group received $3 \mathrm{mg} / \mathrm{kg}$ every two weeks along with a dacarbazine-matched placebo every three weeks, while patients in the chemotherapy group got $1000 \mathrm{mg}$ of dacarbazine per square meter of body-surface area every three weeks along with nivolumab-matched placebo every two weeks. Treatment was provided until the disease progressed or produced a toxic effect. In this study, the Response Evaluation Criteria in Solid Tumors (RECIST) was used to grade tumor response. The primary endpoint was overall survival. Progression-free survival was assessed and determined per the investigator's judgment.

The average length of median overall survival was reported to be 10.8 months for patients treated with dacarbazine; the median overall survival for patients treated with nivolumab was not reached because the overall survival rate for said group was always more than $50 \%$ during the course of the entire study. The rate of overall survival after one year in the nivolumab group was $72.9 \%$, compared to $42.1 \%$ in the chemotherapy group. The average survival time without progression in patients treated with nivolumab was 5.1 months and 2.2 months in those treated with dacarbazine. Patients in the nivolumab group achieved an objective response rate of $40 \%$, while those in the dacarbazine group achieved only $13.9 \%$. Complete responses were noted more in the study group compared to the chemotherapy group. In the nivolumab group, a reduction in tumor burden of at least $30 \%$ was achieved after progression of disease in $8.1 \%$ of patients. In the dacarbazine group, a reduction in tumor burden of at least $30 \%$ was achieved after progression of disease in only $3.8 \%$ of patients. ${ }^{14}$

The subgroups in this study were determined by PD-LI status, negative or positive, assessed through an immunohistochemical assay, which was not relevant in establishing overall survival. If $5 \%$ or more of tumor cells demonstrated cell-surface PD-L1 staining in at least 100 cancer cells, then it was considered to be PD-L1 positive. Within the nivolumab group, neither the positive nor negative PD-L1 subgroup attained median overall survival. With dacarbazine, the average overall survival in the positive PD-L1 subgroup was a little longer. Both subgroups treated with nivolumab had better objective response rates than patients treated with dacarbazine. ${ }^{14}$

There was not a significant difference in adverse events between the experimental group and the control group, although patients in the nivolumab group presented with lesser grade 3-4 adverse events such as diarrhea and increased percentage of alanine aminotransferase. The most common side-effects in the nivolumab group were fatigue, pruritus and nausea, while in the dacarbazine group, they were gastrointestinal and hematological toxicity. There were no deaths related to either study treatment. ${ }^{14}$ 


\begin{tabular}{cccc}
\hline \multicolumn{4}{l}{ Table 6. Nivolumab in previously untreated melanoma without BRAF mutation. ${ }^{14}$} \\
\hline & Primary Endpoint & \multicolumn{2}{c}{ Secondary Endpoints } \\
\hline Overall survival & Progression free survival & Objective Response rate \\
\hline Nivolumab & $72.9 \%$ & $5.1 \mathrm{mos}$ & $40 \%$ \\
\hline Placebo & $42.1 \%$ & $2.2 \mathrm{mos}$ & $13.9 \%$ \\
\hline
\end{tabular}

Progression free survival: patient's disease reaches a plateau and no longer progresses.

Objective response rate: the percentage of patients who had some type of positive response to treatment, including both complete and partial responses

The randomized, controlled, phase III study conducted by Weber et al compares the role of nivolumab versus investigator's choice of chemotherapy (ICC), either dacarbazine, paclitaxel or carboplatin, in treating patients who have progressed with advanced melanoma following anti-CTLA-4 treatment, specifically ipilimumab or ipilimumab and a BRAF inhibitor. ${ }^{15}$ The study involved 272 patients in the nivolumab group and 133 in the ICC group after randomization. The inclusion criteria were age of at least 18 years, confirmed, stage IIIC or IV metastatic melanoma, and in patients with BRAF mutation, disease progression following anti-CTLA-4 treatment and a BRAF inhibitor. The exclusion criteria were metastases of the brain, prior treatment with anti-PD-1 and its ligands, or primary ocular melanoma. Due to not being able to meet study criteria and consent withdrawal, four patients receiving nivolumab and 31 patients receiving ICC were excluded. From the remaining population, those who were qualified for primary assessment of objective response were 120 patients in the study group and 47 in the ICC group. The researchers used RECIST version 1.1 to assess tumor responses. ${ }^{15}$

After treating patients with nivolumab per-protocol for 5.3 months on average, the investigators noted that the median follow-up time of those who attained an objective response was 8.4 months. The principal goal of objective response was achieved in 38 patients in the nivolumab group compared to five in the ICC group. The average length of response was 3.5 months in the ICC group, while it was not reached in the study group. The median time to produce a response with nivolumab was 2.1 months, compared to 3.5 months in the control group. Of the 38 patients who had objective responses with nivolumab, 33 were able to endure treatment without disease advancement. Thirty-one percent of 120 patients in the nivolumab group were able to sustain treatment regardless of progression, which was defined as more than $30 \%$ decrease in the target lesion after initial progression; moreover, $8 \%$ had a one-third decrease in the length of marked lesions. The progression-free survival at six months for patients treated with nivolumab was $48 \%$ and for those treated with ICC was $34 \% .{ }^{15}$ When examining subgroups determined by BRAF status, previous anti-CTLA-4 benefit, or PD-L1 status, it was found that there were more responses, whether complete or partial, in the study group compared to ICC group.

Adverse events occurred in $68 \%$ of patients in the study group, with the most common being fatigue, itching, and diarrhea, and in $79 \%$ of patients in the control group, with nausea, fatigue, and hair loss being the most common. Treatment-related and drug-related grade 3-4 adverse events such as fatigue, anemia, vomiting, and neutropenia were noticed more in the ICC group than in the nivolumab group. Although there were grade 3-4 select adverse events in the nivolumab group, they were reversed by delaying the next dose and using immunosuppressive medicines. Due to the progression of melanoma, $43 \%$ of nivolumab patients and $61 \%$ of ICC patients had to stop treatment. Another reason for ending treatment was drug toxicity, and it affected a small portion of patients in both groups. There were no reported deaths linked to the study treatments. ${ }^{15}$ 
Table 7. Nivolumab versus chemotherapy in patients with advanced melanoma who progressed after anti-CTLA-4 treatment ${ }^{15}$

\begin{tabular}{cccccc}
\hline & \multicolumn{2}{c}{ Primary Endpoint } & \multicolumn{3}{c}{ Secondary Endpoints } \\
\hline \multirow{2}{*}{ Overall Survival } & \multirow{2}{*}{ Objective Response } & $\begin{array}{c}\text { Progression free } \\
\text { survival }\end{array}$ & \multicolumn{2}{c}{ PDL-1 status } \\
\cline { 5 - 6 } & & $31.7 \%$ & $4.7 \mathrm{mos}$ & $77 \%$ & Positive \\
\hline Nivolumab & $12.3 \mathrm{mos}$ & $10.6 \%$ & $4.2 \mathrm{mos}$ & $9.1 \%$ & $13 \%$ \\
\hline Placebo & $8 \mathrm{mos}$ & &
\end{tabular}

Objective response: any positive response to treatment after the start of the study

Progression free survival: patient's disease reaches a plateau and no longer progresses.

PDL-1 Status: an objective biomarker expressed in the tumor, which was used to predict overall survival

Ribas et al performed a randomized clinical trial including 655 treatment-naive patients with unresectable stage IIIC or IV melanoma to assess overall survival, duration of response, safety, as well as progression-free response in patients treated with the monoclonal antibody tremelimumab versus standard chemotherapies temozolomide or dacarbazine. ${ }^{16}$ Progression-free survival (PFS) is defined as the time during and after treatment when the disease reaches a plateau and does not progress. In this study, progression-free survival was assessed at six months. The inclusion criteria were patients 18 years and older with unresectable stage IIIC or IV metastatic melanoma with no prior history of treatment, an Eastern Cooperative Oncology Group performance status of $\leq 1$, serum lactate dehydrogenase of $\leq 2 x$ the upper limit of normal, and adequate renal, hepatic and bone marrow function. Exclusion criteria included patients with brain metastasis or uvula melanoma. In this study, RECIST guidelines were used to evaluate tumor response.

There were a total of 655 patients randomly assigned to the control arm with standard chemotherapy or to the new monoclonal antibody tremelimumab. Patients treated with tremelimumab were administered a $15 \mathrm{mg} / \mathrm{kg}$ dose via intravenous infusion once every 90 days. While patients on dacarbazine were administered $1,000 \mathrm{mg} / \mathrm{m}^{2}$ via intravenous infusion once every 21 days or temozolomide $200 \mathrm{mg} / \mathrm{m}^{2}$ orally on days one through five of a 28 day cycle. ${ }^{16}$ Patients treated with the monoclonal antibody were assessed every 90 days and patients on dacarbazine or temozolomide were assessed every 42 days and 56 days. The average duration of treatment was two to three months. The objective response rate was $10.7 \%$ in the tremelimumab arm and $9.8 \%$ in the standard chemotherapy arm. ${ }^{16}$ Median response duration to treatment was much longer for tremelimumab at 35.8 months versus standard chemotherapy at 13.7 months. After treatment, two- and three-year survival rates were $26.4 \%$ and $20.7 \%$ for tremelimumab and $22.7 \%$ and $17.0 \%$ in patients treated with traditional chemotherapies. Three years after the start of the study, in the final study analysis, median overall survival was 12.6 months for tremelimumab and 10.7 months in the chemotherapy arm. In phase I and II trials, tremelimumab showed longer responses in a portion of the patients with advanced stage melanoma. However, the phase III study, which focused on the overall survival rate and efficacy of tremelimumab, failed to provide an increased survival advantage over treatment with standard-care chemotherapy.

The main side effects noted for tremelimumab included diarrhea, pruritus and rash, yet a small percentage of patients reported more severe side effects including thyroid, hypothalamic or pituitary gland disorders, and adrenal insufficiency. ${ }^{16}$ For the two conventional chemotherapeutic agents, temozolomide or dacarbazine, the most common reported side effects were nausea/vomiting, constipation, and fatigue. Seven treatment-related deaths were recorded for the monoclonal antibody, whereas only one death was recorded in the treatment with standard of care chemotherapies dacarbazine or temozolomide. 
Table 8. Phase III randomized clinical trial comparing tremelimumab with standard of care chemotherapy in patients with advanced melanoma ${ }^{16}$

\begin{tabular}{cccccc}
\hline & $\begin{array}{c}\text { Primary } \\
\text { Endpoint }\end{array}$ & \multicolumn{3}{c}{ Secondary Endpoints } \\
\hline & $\begin{array}{c}\text { Overall } \\
\text { survival }\end{array}$ & $\begin{array}{c}\text { Progression free } \\
\text { survival }\end{array}$ & $\begin{array}{c}\text { Best overall } \\
\text { response }\end{array}$ & $\begin{array}{c}\text { Duration of } \\
\text { response }\end{array}$ & Safety \\
\hline Tremelimumab & 12.6 months & $20.3 \%$ & $10.7 \%$ & 35.8 months & $85 \%$ \\
\hline $\begin{array}{l}\text { Chemotherapy } \\
\text { (Dacarbazine or } \\
\text { Temozolomide) }\end{array}$ & 10.7 months & $18.1 \%$ & $9.8 \%$ & 13.7 months & $28 \%$ \\
\hline
\end{tabular}

Progression free survival (OS): patient's disease reaches a plateau and no longer progresses. Measured at six months after the start of treatment.

Safety (S): percent of patients who experienced an adverse event. Most adverse events were mild grade $1 / 2$ including diarrhea, pruritus, and rash. Grade $3 / 4$ adverse events related to tremelimumab included diarrhea (14\%) and endocrine events $(7 \%)$ such as thyroid or hormone effects. Grade $3 / 4$ adverse events related to chemotherapy included neutropenia $(10 \%)$ and thrombocytopenia $(2 \%)$.

\section{DISCUSSION}

The primary goal of the study conducted by Kirkwood et al was to find the best overall response. ${ }^{10}$ The responses to tremelimumab were considered durable, in that, all patients that had an objective response also sustained their response for more than 170 days after enrollment into the study. As such, tremelimumab has shown durable clinical activity in second-line treatment of patients with surgically-incurable stage III or IV melanoma, suggesting a potential role for tremelimumab in melanoma. Moreover, the study found that an increase in lactate dehydrogenase does not bias against response to therapy, as had been suggested for chemotherapy, and as such, the exclusion of patients from trials based on elevations in LDH does not seem to be appropriate. Analysis of antitumor responses in the study also do not support the current assumption that immunotherapy only benefits the healthiest of patients, while only causing a burden to those with minimal disease. Additionally, it is important to note that Pfizer funded this study's medical editorial assistance and there is a likelihood of publication bias.

Adverse effects of tremelimumab must also be considered, as most (77\%) of the patients developed a treatmentrelated adverse effect, with most of the effects being mild to moderate. Only $5 \%$ of patients discontinued the trial due to treatment-related adverse effects, and only two $(0.8 \%)$ treatment-related deaths occurred. ${ }^{10}$

More studies must be performed in order to identify the specific patient population which will respond to tremelimumab, and to determine the early indicators for later response to therapy, thus improving the therapeutic index and allowing for other agents or combinations for those who fail to respond to tremelimumab. Currently, studies using combinations of tremelimumab with vaccines and with IFN- $\alpha$ are in progress. ${ }^{10}$

The study conducted by Eggermont et al showed significantly increased recurrence-free survival with use of ipilimumab in patients with completely resected high-risk stage III melanoma. ${ }^{11}$ Recurrence-free survival was determined by calculating the amount of time between the date of randomization and either first recurrence of disease or death, whichever occurred first. Based on a phase II trial, the dose of $10 \mathrm{mg} / \mathrm{kg}$ was chosen, as it had shown the best results, however, this dose was markedly higher than the approved dose of $3 \mathrm{mg} / \mathrm{kg}$ for treatment of patients with advanced melanoma. In the EORTC 18991 trial, adjuvant pegylated interferon was compared to observation. This study showed the importance of studying microscopic versus macroscopic nodal involvement, as well as the presence or absence of ulceration of the primary melanoma. It was suggested that lymph nodes with tumors of microscopic size may have a greater benefit than patients with larger palpable lymph nodes. Similarly, in Eggermont et al's study, ipilimumab showed that this held true, however, there was still a substantial benefit in the patients with palpable nodes as well as patients with ulcerated melanoma. In addition, interferon also had a substantial effect on patients with nodal involvement. As such, it was noted that ulcerated melanoma is a "separate biological entity". ${ }^{11}$ Additionally, it is important to note that Bristol-Myers Squibb, GlaxoSmithKline, Roche, Merck, and Agmen served on the advisory board and funded this study, increasing the likelihood of bias. 
The adverse effect profile for ipilimumab must also be considered, as nearly $40 \%$ of the patients discontinued treatment before the completion of the initial dosing period, that is, before the maintenance period; the reason behind this remains to be investigated. While knowing if this high adverse effect profile is due to longer drug exposure or greater sensitivity remains speculative, in a pooled analysis of studies using a dose of $10 \mathrm{mg} / \mathrm{kg}$ in patients with advanced melanoma, most immune-related manifestations resolved within four to six weeks. The drug-related fatalities were also alarming. Although great care was taken to monitor the patients closely in addition to meticulous instruction of the patients, $1 \%$ of the patient population in the study died, due to drug-related causes after discontinuing ipilimumab. ${ }^{11}$ It is unknown if the treatment-related deaths could have been prevented if the complications had been managed differently.

The definitive value of distant metastasis-free survival and overall survival endpoints have yet to be defined, and the risk-benefit ratio of adjuvant ipilimumab at the dose tested requires further evaluation. Additional studies must also be performed to provide additional insight on the benefits of higher dose ipilimumab versus the standard dose used for unresectable metastatic disease, and to discover if either dose of ipilimumab truly improves outcomes or is less toxic than high-dose interferon. ${ }^{11}$

The clinical studies involving ipilimumab $10 \mathrm{mg} / \mathrm{kg}$ in patients with melanoma used data from programs at Italian centers known as an expanded access programs. Altomonte et al and Di Giacomo et al evaluated clinical activity and safety of the monoclonal antibody in patients with advanced melanoma disease and pretreated patients with advanced disease in a daily practice setting. ${ }^{12,13}$ This type of study was important to include because it is more indicative of ipilimumab safety and efficacy in the typical setting for day-to-day patients and not just within the parameters of clinical trials.

After thorough analysis of heavily pretreated patients with advanced melanoma, Altomonte et al concluded that the expanded access program suggested that the clinical activity and safety profile of ipilimumab was actually similar to the results found in clinical trials. ${ }^{12}$ The author states that the safety profile, adverse effects, tumor assessment and response, and overall survival rates were actually consistent with the results reported in previous phase II and III clinical trials. ${ }^{12}$

Most adverse events were consistent with previous trials and consisted mainly of dermatological and gastrointestinal occurrences. Sixty-one percent of patients experienced adverse effects related to their treatment, but no deaths were related to their therapy. Interestingly, there was a decrease in the amount of reported high-grade adverse, events which the authors believe may have been due to the "retrospective" nature of this study. They also point out that there is a decrease in the requirement for intervention in incidents of bowel perforation, which may be due in large part to better management and the advent of more clear algorithms for proper management. Overall, the use of established algorithms for the proper management of adverse events and the ability to anticipate specific side effects of ipilimumab has led to a more manageable safety profile, which demonstrates that ipilimumab may be safely administered and managed by community-based physicians in a daily clinical setting. ${ }^{12}$

An assessment of tumor response rates at week 12 demonstrated a $9 \%$ objective response. Altomonte et al note that beyond week 12 an increase in the number of patients that achieved an objective response was observed, possibly due to the fact that the body requires time for the immune response to affect tumor size after stimulation by monoclonal antibodies. ${ }^{12}$ Overall survival was observed to be seven months. The median survival rates for other clinical trials have been shown to be approximately 10 months, which is different from the seven months median overall survival observed in this study. The authors note that it is important to take into consideration that the patients who participated in this study had a very poor prognosis and significantly advanced disease as classified by their $\mathrm{M}$ status which defines severity with respect to metastasis. ${ }^{12}$ Other studies have highlighted poor performance status, visceral disease, brain metastases, and $\mathrm{M}$ status as factors that have a statistically significant effect on prognosis. At least two years of survival was observed by one-fifth of patients with approximately $17 \%$ of patients alive at three years. Although this study proved the efficacy of ipilimumab in treating patients with melanoma, more studies are needed to establish the proper dose for maximum treatment benefit. The expanded access program for this study was sponsored by BristolMyers Squibb. Additional funds were provided to support editorial and writing assistance by StemScientific through the Bristol-Myers Squibb company. ${ }^{12}$

Di Giacomo et al also found ipilimumab to be a viable treatment option for malignant melanoma in previously-treated patients experiencing a progression of their disease. ${ }^{13}$ The expanded access program showed that a large number of 
the patient population benefited from the treatment and resulted in long-term survival similar to that observed in phase II clinical trials. It is of note that this study has a small sample size of 27 patients, which limits it applicability to clinical use. Two case studies were included within this article, however their findings were not specifically reported since case studies were excluded in our analysis. ${ }^{13}$

Objective responses with disease control was achieved in $22 \%$ of patients with a median overall survival of 9.6 months, one year survival rate of $34.8 \%$ and two year survival rate of $23.5 \%$. In addition, the safety profile of ipilimumab in this program was found to be substantially similar to that seen in previous phase II and III clinical trials. Treatment was associated with generally mild immune or mechanism-related side effects that were manageable and reversible in most cases, which also parallels similar results in clinical trials. These findings are important and helpful for community physicians using ipilimumab in daily practice, since it illustrates that following management protocols for these adverse events facilitates the use of monoclonal antibodies for this indication. The author suggests that strict adherence to this treatment protocol with this monoclonal antibody will provide a strong contribution to allow safe use of this agent in daily clinical practice. ${ }^{13}$

Furthermore, a sizeable proportion of the patients in the expanded access program experienced a durable clinical response, including benefits to long-term survival. Disease control with ipilimumab also followed a pattern slightly different than that seen with chemotherapy. It was observed that disease control may be delayed or can be seen following further treatment after disease progression, posing the possibility that continued treatment may benefit patients with stable or progressive disease. Di Giacomo et al also mention that a unique immune-related response criteria to evaluate clinical response may be a more adequate method of evaluating tumor response than WHO or RECIST criteria. ${ }^{13}$ With these observations and conclusions, patients and treating physicians will have a greater awareness of the clinical response patterns and side effects related to ipilimumab. ${ }^{13}$

This study received editorial and writing assistance from StemScientific funded by Bristol-Myers Squibb company. Through this support, the expanded access program was able to provide evidence to show that ipilimumab is an effective and safe treatment option to use in daily practice for metastatic melanoma. These clinical responses also show that, even with failed prior treatment options, disease progression can be stopped with this monoclonal antibody. ${ }^{13}$

The results of the experiment performed by Robert et al indicate that an overall survival rate was attained more in patients treated with nivolumab compared to those treated with dacarbazine. ${ }^{14}$ Death risk for patients in the nivolumab group was lowered by $58 \% .{ }^{14}$ The benefit of survival was steady among all subgroups. The survival rate at one year in the nivolumab group is greater than that in the dacarbazine group, and is in accordance with previous study results. The reason behind choosing dacarbazine as the chemotherapy drug was that, until recent times, it was the primary treatment option for those with melanoma void of BRAF mutation. A greater median survival of 10.8 months was noted in the dacarbazine group, most probably due to the fact that ipilimumab was given to $38 \%$ of patients in this group following cessation of the study treatment. The response to treatment was higher in the nivolumab group with 16 patients achieving complete responses, 68 partial responses and 35 stable disease, compared to two complete responses, 27 partial responses, and 46 stable disease in the ICC group.

From the current research, it was found that a long-lasting, fast, and an increased rate of response was achieved in those who were given nivolumab. All these results regarding nivolumab are consistent with previous research which has shown similar clinical effects, irrespective of BRAF mutation type. Furthermore, as noted in preceding trials, the safety profile was good and the number of toxic effects were minimal. When choosing patients for therapy with nivolumab, the status of PD-L1 by itself does not appear to be helpful, and therefore its role in prognosis needs to be understood in future research. The funds needed for this study were distributed by Bristol-Myers Squibb pharmaceutical company. ${ }^{14}$

The study done by Weber et al found that more patients treated with nivolumab, an anti-PD-1 antibody, had complete objective responses compared to those treated with ICC..$^{15}$ However, a major variation in survival without progression was not statistically identified, most probably due to "the imbalance in distribution of adverse prognostic factors in favor of the ICC group, immaturity of the data, and the false-positive disease progression in the nivolumab group." ${ }^{15}$ In the nivolumab group there were four complete responses, 34 partial responses, and 28 patients with stable disease compared to the ICC group with zero complete responses, five partial responses, and 16 stable disease. The data and results achieved with nivolumab in the present experiment are in agreement with preceding trials. Nivolumab has been 
accepted by the United States Food and Drug Administration for patients who have disease advancement following ipilimumab due to prolonged responses. The result of lowered tumor development past RECIST 1.1-defined progression in $8.3 \%$ of patients in the nivolumab group indicates that nivolumab might have a higher potential for benefit, and could be the reason why there was not a noteworthy difference in survival without progression. Regardless of the type of BRAF mutation, nivolumab has been found to be effective as noted in previous studies. ${ }^{15}$

One drawback to this study lies in the analysis of data, where it was noted that 22 patients in the ICC group withdrew consent, while only one patient in the study-drug group withdrew consent. The authors discussed that this large number of withdrawals in the control group may have been due to patients seeking alternative, more advanced treatments, such as other monoclonal antibodies. These patients may have been well-informed regarding the advent of monoclonal antibodies and had resources affording them this option. The authors attribute the underlying reason for this to be that the study was not an open-label, non-crossover study design. Even though using immune-related response criteria could have produced better results in this study, this criteria is not verified by regulatory agencies, and therefore could not be utilized. It was noted that there was more general safety with nivolumab compared to chemotherapy, and adverse effects were controlled by drugs and dosage changes. This study was also funded by the Bristol-Myers Squibb company. The authors who held leadership roles during this research were Jeffrey $S$ Weber, and James Larkin. ${ }^{15}$

Overall results in Ribas et al's study comparing the monoclonal antibody tremelimumab to standard chemotherapy with dacarbazine or temozolomide concluded tremelimumab was minimally effective in overall survival. ${ }^{16}$ Tumor response rates to treatment showed similar progression in reduction of size. Since the duration of responses were significantly improved, the trial confirms the benefit from treatment with tremelimumab in a certain subset of patients.

The results of this study are further unsupported due to the recent change in the standard of care for melanoma. Now, the traditional chemotherapy drugs are less commonly used and other new monoclonal antibodies such as ipilimumab and the BRAF inhibitor, vemurafenib, have been approved for use. In a single-arm phase II study of ipilimumab at 10 $\mathrm{mg} / \mathrm{kg}$ every three weeks, proved to demonstrate similar efficacy to the tremelimumab phase II trial. Furthermore, patients with previously-treated melanoma had a greater overall survival when treated with a lower monthly dose of ipilimumab as opposed to monotherapy with the gp100 vaccine. Additionally, a phase III clinical trial of ipilimumab recognized the enhanced efficacy of combination therapy (ipilimumab + dacarbazine) versus monotherapy (ipilimumab) in patients with metastatic melanoma.

The authors also recognize the possibility of patient selection enriching tumor responses and improved outcomes in the control arm, which decreased the survival difference between treatments with tremelimumab in comparison to chemotherapy. Also of noteworthy mention in this study, is the exclusion of patients with serum lactate dehydrogenase $\leq 2$, as similar studies did not have this exclusion factor. During the course of this study, the drug of choice for advanced melanoma changed. Consequently, some patients in the control arm who were under treatment with dacarbazine or temozolomide chose to enroll in concurrent studies, where they were given add-on therapy with the monoclonal antibody ipilimumab. Five patients admitted to enrolling into this dual therapy; however, Ribas et al recognizes that this is probably an underestimate of the number of patients who enrolled in additional treatment with ipilimumab.

Several other inconsistencies in this study may suggest unreliable results. One confounding variable includes the reduced number of original patients in each phase of the trial. Eight months after the start of the study, 340 deaths had occurred secondary to metastasis of patients' advanced melanoma. Two years later, 534 total deaths had occurred which was $82 \%$ of the original population deceased. This left only 131 participants to follow through the end of the study. Additionally, at the start of the study, one inclusion criteria for patients was a serum lactate dehydrogenase level $\leq 2 x$ the upper limit of normal with adequate bone marrow, hepatic, and renal function. When the study began, it was noted about $5 \%$ of patients had $>2 x$ upper limit of normal for $L D H$. The variability in route of administration as well as dose and duration of treatment varied between both the monoclonal antibody and standard of care chemotherapy, but also between dacarbazine and temozolomide, making it hard to compare the therapies. Due to these differences in treatment outcomes, further studies need to be performed before adequate conclusions can be obtained. Funding for this study was provided by several large scale pharmaceutical companies including Bristol-Myers Squibb, Pfizer, GlaxoSmithKline, Genta, MSD, Synta, SOBI, Novartis, and Roche; all of which may result in unintentional biases. Additionally, the main leadership roles in this study were all held by individuals employed by Pfizer including Margaret Marshall, Jesus Gomez-Navarro, Bo Huang, and Dmitri Pavlov. 


\section{CONCLUSION}

Melanoma is a serious condition that requires research of modern therapy agents to help improve patient's quality of life during treatment and prognosis. In this paper, various studies that analyze the safety and efficacy of monoclonal antibodies, which have recently been upgraded as first-line for the treatment of melanoma, were evaluated. As previously mentioned, chemotherapy and immunotherapy consisting of IL-2 was first-line, but due to its side effect profile and inadequate efficacy, monoclonal antibodies are now being used as first-line treatment. The goal of this paper was to validate the use of monoclonal antibodies as first line treatment.

Of the seven studies evaluated, six demonstrated that monoclonal antibodies do have a prominent role in the treatment for advanced-stage melanoma. Three studies directly compared the use of monoclonal antibodies to chemotherapy. The studies conducted by Robert et al and Weber et al showed a statistically significant advantage of monoclonal antibodies over chemotherapy. The third study conducted by Ribas et al failed to demonstrate a statistically significant survival advantage over chemotherapy.

It is noteworthy that inconsistencies existed among the primary and secondary endpoints evaluated in each study, which may affect the overall interpretation of data. And, although each study had similar inclusion and exclusion criteria, variation among criteria did exist. For example, non-metastatic vs. metastatic inclusion/exclusion criteria and the use of previous systemic therapy with other therapeutic agents varied among each study. Regardless of these inconsistencies, the studies demonstrated monoclonal antibodies remained efficacious.

Another inconsistency is the method the various studies used to evaluate tumor response. RECIST and WHO were the primary modalities used to measure tumor response. Monoclonal antibodies have a mechanism of action which differs from most other systemic treatment used to treat tumors. Monoclonal antibodies work by eliciting an immune response from the patient's body, which is directed to kill the tumor cell. RECIST and WHO were not developed to evaluate tumor response to this mechanism. Therefore, when evaluated using these methods, monoclonal antibodies may not have the chance to show their true response, especially when using these methods to compare them directly to chemotherapy. Methods to evaluate immune-related tumor response are currently being developed, but none have been evaluated by regulatory agencies and are therefore not used in these studies. ${ }^{15}$

All seven studies demonstrated that monoclonal antibodies have a manageable adverse event profile, especially when compared to previously used immunotherapies. Grade I and II adverse events make up the majority of side effects seen in all seven studies, most commonly fatigue, pruritus nausea and diarrhea. Kirkwood et al ${ }^{10}$ had two patient $(0.8 \%)$ deaths, Eggermont et al had five patients $(1 \%)$ deaths and Ribas et al had seven patient deaths directly related to treatment with monoclonal antibodies. Di Giacomo et al reported one patient death categorized as possibly related to the treatment with monoclonal antibodies; however, this patient had further metastasis and other comorbidities. Ribas et al also reported one death directly related to treatment with chemotherapy. When directly compared to chemotherapy, the monoclonal antibodies had a better adverse event profile. ${ }^{14-16}$ The studies conducted by Altomonte et al and Di Giacomo et al showed that the adverse event profile of monoclonal antibodies can be managed using proper treatment algorithms at typical treatment centers and does not require the use of special facilities and physicians even with patients with more advanced disease and comorbidities.

Further investigation is warranted to determine which of the monoclonal antibodies is best for the treatment of melanoma and how combination therapy of multiple monoclonal antibodies, concurrent chemotherapy, vaccines, and enzyme inhibitors affect treatment outcomes.

\section{DISCLOSURE}

The authors report no conflict of interest, affiliation, or financial partnerships with any of the cited authors or pharmaceutical companies mentioned in this review.

\section{REFERENCES}

1. 10 Must-know 2015 global cancer facts. American Cancer Society. 2015. Available at: http://www.cancer.org/research/acsresearchupdates/more/10-must-know-2015-global-cancer-facts. Accessed December 2, 2015. 
2. What are the key statistics about melanoma skin cancer. American Cancer Society. 2015. Available at: http://www.cancer.org/cancer/skincancermelanoma/detailedguide/melanoma-skin-cancer-key-statistics. Accessed December 2, 2015.

3. What are the survival rates for melanoma skin cancer, by stage. American Cancer Society. 2015. Available at: http://www.cancer.org/cancer/skincancer-melanoma/detailedguide/melanoma-skin-cancer-survival-rates. Accessed December 2, 2015.

4. Crosby T, Fish R, Coles B, Mason M. Systemic treatments for metastatic cutaneous melanoma. Cochrane Database of Systematic Reviews. 1996;2000(2). doi:10.1002/14651858.cd001215.

5. Stevens M, Hickman J, Langdon S, et al. Antitumor activity and pharmacokinetics in mice of 8-carbamoyl-3-methylimidazo[5,1-d]-1,2,3,5-tetrazin-4(3H)-one (CCRG 81045; M \& B 39831), a novel drug with potential as an alternative to dacarbazine. Cancer Res. 1987;47(22):5846-52.

6. Middleton M, Grob J, Aaronson N, et al. Randomized phase III study of temozolomide versus dacarbazine in the treatment of patients with advanced metastatic melanoma. J Clin Oncol. 2000;10(1):158.

7. Atkins M, Lotze M, Dutcher J, et al. High-dose recombinant interleukin 2 therpay for patients with metastatic melanoma: Analysis of 270 patients treated between 1985 and 1993. J Clin Oncol. 2000;17(7):2015.

8. Sosman J. Overview of the management of advanced cutaneous melanoma. UpToDate. 2015. Available at: $\mathrm{http}: / / w w w . u p t o d a t e . c o m / c o n t e n t s / o v e r v i e w-o f-t h e-m a n a g e m e n t-$ ofadvancedcutaneousmelanoma? source=search_result\&search=treatment+of+advanced+cutaneous+melanoma\& selectedTitle=9 150. Accessed December 2, 2015.

9. Gennigens C, Collignon J, Jerusalem G, Rorive A, Sautois B. Therapeutic monoclonal antibodies in hematooncology. Rev Med Leige. 2009;64(5-6):264-7.

10. Kirkwood J, Lorigan P, Hersey P, et al. Phase II Trial of Tremelimumab (CP-675,206) in Patients with Advanced Refractory or Relapsed Melanoma. Clinical Cancer Research. 2010;16(3):1042-8. doi:10.1158/1078-0432.ccr-092033. [PMID: 20086001]

11. Eggermont M, Chiarion-Sileni V, Grob J-J, et al. Adjuvant ipilimumab versus placebo after complete resection of high-risk stage III melanoma (EORTC 18071): a randomised, double-blind, phase 3 trial. Lancet Oncol. 2015;16:522-30. [PMID: 25840693]

12. Altomonte M, Di Giacomo A, Queirolo P, et al. Clinical experience with ipilimumab $10 \mathrm{mg} / \mathrm{kg}$ in patients with melanoma treated at Italian centres as part of a European expanded access programme. J Exp Clin Cancer Res. 2013;32(1):82. doi:10.1186/1756-9966-32-82. [PMID: 24423086]

13. Di Giacomo A, Danielli R, Calabrò L, et al. Ipilimumab experience in heavily pretreated patients with melanoma in an expanded access program at the University Hospital of Siena (Italy). Cancer Immunology, Immunotherapy. 2010;60(4):467-77. doi:10.1007/s00262-010-0958-2.

14. Robert C, Long GV, Brady B, et al. Nivolumab in previously untreated melanoma without BRAF mutation. N Engl J Med. 2015;372:320-30. [PMID: 25399552]

15. Weber JS, D'Angelo SP, Minor D, et al. Nivolumab versus chemotherapy in patients with advanced melanoma who progressed after anti-CTLA-4 treatment (CheckMate 037): a randomized, controlled, open-label, phase 3 trial. Lancet Oncology 2015;16(4):375-84. [PMID: 25795410]

16. Ribas A, Camacho LH, Millham R, et al. Phase III randomized clinical trial comparing tremelimumab with standard of care chemotherapy in patients with advanced melanoma. Journal of Clinical Oncology. 2013;31(5):616-22. [PMID: 23295794] 